\title{
The limit problem of the Patlak-Keller-Segel-Stokes system in scalling critical space
}

\author{
Minghua Yang ${ }^{1}$ and Yanchang $\mathrm{Han}^{2}$ \\ ${ }^{1}$ Jiangxi University of Finance and Economics, Nanchang 330032, P.R. China \\ ${ }^{2}$ South China Normal University
}

April 26, 2021

\begin{abstract}
In this paper, we consider a singular limit problem of Cauchy problem for Patlak-Keller-Segel equation coupled with Stokes equation in scalling critical space. Precisely, by taking advantage of a coupling structure of equations and using a scale decomposition technique, it is shown that when the relaxation time parameter $\varepsilon-[?]$, a solution of Patlak-Keller-Segel system coupled with nonstationary Stokes equation converges to that of Patlak-Keller-Segel system coupled with stationary Stokes equation in the critical Fourier-Besov space under certain conditions.
\end{abstract}

\section{Hosted file}

20201007YS-stablility.pdf available at https ://authorea.com/users/410195/articles/519661-thelimit-problem-of-the-patlak-keller-segel-stokes-system-in-scalling-critical-space

\section{Hosted file}

20201007YS-stablility. tex available at https ://authorea.com/users/410195/articles/519661-thelimit-problem-of-the-patlak-keller-segel-stokes-system-in-scalling-critical-space 